\title{
Shenaz Patel : entre conscience environnementale, couleur locale et histoire
}

\author{
Shenaz Patel: Between Environmental Consciousness, \\ Local Colour and History
}

\author{
Sonia Dosoruth \\ Université de Maurice, Maurice
}

Résumé : Shenaz Patel, auteure mauricienne, nous introduit à la cause environnementale dans les albums intitulés La toile bleve (2010) et Le bestiaire mauricien (2016) tout en mettant en exergue la couleur locale. À travers la co-écriture des bandes dessinées Histoire de Maurice (TI et T2) avec Jocelyn Chan Low, elle souhaite promouvoir l'histoire de l'île Maurice.

Si la dynamique de l'écriture fait entendre des sonorités exquises pour un habitué, ce sera à travers la représentation picturale, accompagnée par son texte, que I'histoire et la cause environnementale semblent s'exprimer le plus. Le fait que la notion de frontière-démarcation soit réinventée par une sorte de chevauchement de territoires, permet à une certaine identité littéraire de se former.

Mots-clés : frontières, environnement, animal, homme, histoire.

Abstract: Mauritian writer Shenaz Patel is environmentally conscious in the graphic novels La toile bleue (2010) and Le bestiaire mauricien (2016). By further co-writing Histoire de Maurice (T1 et T2) with Jocelyn Chan Low, Patel shows the importance of knowing about and delving into a nation's history. Patel also allows local colour to express itself.

The art of convincing the reader on the above is emphasized through the graphic novels, which send a clear picture of the themes analysed. It is without difficulty that the blurred demarcations of the themes developed help to gaze through the spirit of the literary identity that comes to light.

Keywords: borders, environment, animal, man, history.

L'exubérante richesse de la nature de l'île Maurice subit des bouleversements causés par le besoin pressant de l'homme d'exploiter les espaces naturels. Dans ses bandes dessinées intitulées La toile bleve (2010) et Le bestiaire mauricien (2016), Shenaz Patel, auteure mauricienne, qui a également écrit Le portrait Chamarel (2002), Sensitive (2003) ou encore Le silence des Chagos (2005), nous renseigne sur les dégradations irréversibles causées par l'homme à la faune et la flore mauriciennes. Dans les deux premiers ouvrages, nous apprenons, d'une part, ce qu'une enfant pense de son 
environnement et des méfaits de la pollution et, d'autre part, on nous explique l'origine de certains animaux locaux. Au-delà de la visée didactique de l'auteure, cette dernière rend également accessible l'histoire de l'île Maurice en la rendant moins complexe. Aussi invite-t-elle prioritairement le jeune lecteur, dans Histoire de Maurice (vol.1) - une bande dessinée historique -, à prendre conscience de la dimension esclavagiste que l'île a connue, même si l'auteure a elle-même expliqué qu'il s'agit d'un "graphic novel qui s'adresse à tous les âges' ". Le deuxième tome mettra quant à lui l'accent sur les figures qui ont marqué l'histoire de l'île ainsi que sur les différents peuples qui se sont installés sur l'île Maurice.

Vivons-nous les derniers jours d'une végétation primitive qui régresse de jour en jour? Les perturbations du milieu lagunaire et corallien causées par les déchets, sonnentelles le glas des zones littorales de l'île ? Cette étude abordera, dans un premier temps, la mise en discours de l'environnement, dans une sélection de textes de Shenaz Patel. Dans un deuxième temps, nous verrons comment Patel valorise la couleur locale. Finalement, nous analyserons I'histoire comme véhicule du savoir dans la bande dessinée, ce qui nous permettra de déterminer si le cadre du savoir historique se limite à un espace défini.

\section{Une mise en discours de l'environnement}

La toile bleve (2010) est un album qui captive dès le premier regard grâce à la couleur bleve qui incarne le bleu de la mer. Cet album est narré par une jeune enfant qui découvre les déchets que rejette la mer. Alors qu'elle pose des questions d'enfant des interrogations normales de la plupart des enfants de son âge - il y a une autre voix narrative qui se fait aussi entendre : il s'agit de celle des animaux de la mer. L'album, dans ses représentations graphiques ainsi que dans son discours narratif, nous donne à voir ce que Sandra L. Beckett reprend dans son livre Crossover Picturebooks: A Genre For All Ages (à travers une citation de Ruy-Vidal), à savoir que: "There is no literature for children. There is literature ॥. Dans la bande dessinée La toile bleve, c'est à travers une teinte d'humour mêlée à un discours des complexités qui sous-tendent la société d'aujourd'hui que Shenaz Patel aborde les perturbations du milieu lagunaire. L'auteure s'attaque au problème environnemental qu'est la pollution des mers et des océans. La préface signée Shenaz Patel et Joëlle Maëstracci l'illustratrice - consiste en une phrase qui se termine par des points de suspension, et qui nous est présentée sous la forme de deux vagues abstraites. En effet, le fond bleu pourrait se référer à l'océan, tandis que les deux lignes typographiées à l'encre blanche ressemblent étrangement à la forme poétique du calligramme. L'incipit de I'histoire montre « [U] ne petite fille (...) assise, face à la mer » (Patel, 2010, p. 8). Elle est, de surcroît, « seule» (Patel, 2010, p. 8), un personnage en marge du récit qui sera raconté, comme s'il s'agissait d'un épilogue malheureux. Cette solitude, qui fait partie du périple existentiel, et qui, souvent, manifeste ses pouvoirs dévastateurs, incarne dans cette bande dessinée un aspect inspirateur qui pousse le personnage à se replier sur lui-même. La fille contemple la mer bleve (de l'espoir ou de l'infini) et "espère ", "sans trop savoir qui, sans trop savoir quoi » (Patel, 2010, p. 8). Dans ce texte, le décor semble posséder une sonorité musicale ${ }^{2}$. Patel démontre comment « [l] e soleil fait le

\footnotetext{
I Shenaz, P. (30 avril 2017). Cessons de nous mentir sur notre Histoire. Interview réalisé par Fabrice Aquilina, Lexpress. www.lexpress.mu [30/04/2017].

2 « Elle attend et se dit qu'elle aimerait/ voir d'animer la GRANDE toile bleue qui s'étend devant elle/ Mais rien ne bouge/ Le soleil fait le dos rond » (Patel, 2011, p 9).
} 
dos rond ॥ (Patel, 2010, p. 9), expression qui fait référence à l'acte de subir des critiques sans réagir, ce qui tend vers l'interprétation première des problèmes environnementaux (avec un soleil - une planète qui donne la vie - qui se courbe, assailli par tant d'ennemis de l'environnement). Notons, par ailleurs, le statisme du décor composé de deux sujets fixes: le soleil et la mer. Par extension, les vagues abstraites de l'épilogue pourraient même s'apparenter à des tsunamis, mot japonais composé de deux racines : tsu, qui signifie port ou rade, et nami, qui signifie vague, ou tout simplement le glyphe qui représente l'eau (à savoir une ligne ondulée qui fait miroiter une cohérence plurielle), accentuant parfaitement la poétique de Patel. En effet, nous voyons ensuite après, l'apparition d'un crocodile qui convoite une "énorme barbe à papa » (Patel, 2010, p. 10), tout en étant sur un nuage:

\begin{abstract}
Une tortue renversée [qui] lorgne un lapin aux oreilles dentelées (...) Une vache aux pis gonflés de pluie [qui] divague en traînant la patte (...) Plus loin, un serpent sans queve ni tête [qui] digère un chapeau trompette (...) Une chenille [qui] s'effiloche en ronds de fumée blanche» (Patel, 2010, pp. 1113).
\end{abstract}

Cette mise en discours de l'environnement se précise lorsque la petite fille s'ennuie du statisme de ce décor. L'enfant parvient donc à se rapprocher de l'universel, d'une part, d'un point de vue esthétique, mais également, et d'autre part, d'un point de vue performatif (par une action individuelle ou en groupe) pour altérer la présence de ce qui constitue l'universalité réelle. C'est à cet instant qu'elle décide de « remettre en place » (Patel, 2010, p. 31) "le morceau de toile bleve » (Patel, 2010, p. 14), ce qui permettrait symboliquement à la toile de se débarrasser de ses déchets pour retrouver sa pureté d'origine. Notons, une fois de plus, le calligramme qui se forme avec l'acte de tirer cette toile. Le jeune personnage va, juste après, marcher « jusqu' à atteindre une corde à linge tendue entre deux poteaux "), où les deux poteaux vont bientôt incarner les deux extrémités possibles, un peu à la manière des pôles. En étendant ce linge particulier sur la corde, c'est à un monde merveilleux que l'on est convié, pour la deuxième fois (après les animaux qui vivent sur les nuages), par des :

flocons cotonneux gorgés de cristaux scintillants [qui] coulent le long de la toile et s'égouttent sur le sol (Patel, 2010, p. 17).

C'est à cet instant que la magie opère. On pourrait ici rapprocher cette description de ce que Max Bense précise dans Théories générales des textes. Max Bense pense en effet que l'information sémantique d'un message linguistique n'est pas nécessairement le message de l'œuvre littéraire. En effet, même si l'on perçoit la manière à travers laquelle l'enfant - un peu par curiosité - étend le linge, ce que nous verrons par la suite est que le réel message de l'auteure est très profond. L'enfant met à l'index l'homme qui, par sa course effrénée à la croissance, a largement contribué à dégrader les écosystèmes côtiers. II y a dans cette écriture patelienne comme un appel à une écogenèse qui inviterait l'enfant à prendre conscience d'un aspect plus complexe, à savoir l'aspect éthique de la problématique environnementale. Chaque image véhiculée dans la représentation graphique ainsi que dans les couleurs choisies met en lumière soit la beauté de la nature soit les périls environnementaux provoqués par l'urbanisation, l'industrialisation et le manque de civisme. II est, à ce sujet, intéressant de se pencher sur la définition de l'écocritique proposée par Cheryll Glotfelty : 
Qu'est-ce que l'écocritique ? Dit simplement, l'écocritique est l'étude du rapport entre la littérature et l'environnement naturel. Tout comme la critique féministe examine le langage et la littérature d'une perspective consciente du genre [gender], tout comme la critique marxiste apporte une conscience des rapports de classe et des modes de production à sa lecture des textes, l'écocritique amène une approche centrée sur la Terre aux études littéraires (Glotfelty, 1996, p. 18).

Ces divers rapprochements nous incitent à lire les textes ayant trait à l'environnement à travers une perspective poétologique afin de mieux circonscrire l'esthétique écocritique dominante dans les bandes dessinées étudiées.

Lorsque la petite fille « jette la toile en travers de la corde " (Patel, 2010, p. 17), il tombe des «flocons cotonneux gorgés de cristaux scintillants», ce qui, sur le moment, la pousse à demander à sa grand-mère « de lui tricoter un pull » (Patel, 2010, p. 17). Cela pourrait métaphoriquement faire référence aux trésors que la mer offre à I'homme comme les " cristaux scintillants » qui peuvent être utilisés au quotidien pour " tricoter un pull». Or, l'intention de l'auteure semble double : le message est adressé à l'enfant comme à l'adulte. Comme en épilogue à un livre ou à un film, on assiste à la chute d'autres "trésors " (Patel, 2010, p. 18) tels que des " oiseaux à bec canne-àpêche " (Patel, 2010, p. 18), des "fragments d'arc-en-ciel» qui "mélangent leurs couleurs $"$ (Patel, 2010, p. 19). Nous assistons au mécontentement de ceux qui ont été arrachés à leur mère nourricière et qui subissent la domination tentaculaire de l'homme. Les contestations de la voix narrative vont soudain s'arrêter lorsque, " [d] ans un soubresaut, la toile bleve vomit une avalanche " (Patel, 2010, p. 26), donnant lieu à un calligramme bien désordonné, comme s'il décrivait minutieusement l'état d'âme de cette vague. Eł comme le précise Gao Xingjiang (2010, p. 6),

La détresse existentielle de l'homme contemporain est ainsi reliée, par un fil invisible, avec la détresse de l'auteur et de la littérature elle-même...

On vit cette description si intensément qu'on ne peut exclure la part volontaire de l'auteure d'y laisser sa marque.

Patel conçoit son œuvre de manière à interpeller, à susciter de l'intérêt pour les empreintes laissées par la société. Alors que la petite fille, qui, ayant pris peur des vociférations du « public », décide de descendre la toile, elle n'y parvient pas. Et, " La toile bleve régurgite enfin les restes de sa cargaison », à savoir : " un lavabo, un vélo, un paquebot, des pneus» et "une auto" (Patel, 2010, p. 35). Les préoccupations écologiques sont ici de grande envergure puisqu'elles recèlent un discours axiologique qui repose sur deux postulats ; l'une mettant en évidence la surconsommation et l'autre liée au non-recyclage de certains déchets. Dans un monde utopique, la toile bleve s'étant débarrassée des polluants qui la rendaient " malade », " rattrape ses oiseaux, ses nuages, ses bigorneaux, ses coraux, ses poissons, ses oursins et se laisse couler le long de la pente" (Patel, 2010, p. 36) avant de regagner sa position initiale sous les applaudissements de la foule. Et la corde devient le symbole de l'horizon tout en laissant un pli « en son milieu » (Patel, 2010, p. 40). Cette histoire se termine par une forme de mise en garde en indiquant que s'il advient que l'on contrarie la toile bleve, elle se glissera et, « (...) ils ne seront pas trop de la terre entière pour aller l'en tirer" (Patel, 2010, p. 9). Ce faisant, l'auteure nous invite à prendre conscience de l'importance de l'environnement et, plus symboliquement ainsi que plus intérieurement, à nous transformer. Alain Bourque précise : 
Le réchauffement des températures ou toute modification aux paramètres climatiques ne peut faire autrement qu'engendrer des impacts sur l'environnement et l'activité socio-économique. En effet, les "secteurs" comme l'agriculture, la foresterie, les écosystèmes, les infrastructures, les pêches, la gestion de l'eau, le tourisme, l'activité économique, la production et la demande d'énergie, en sont tous qui se sont ajustés aux paramètres climatiques historiques (Bourque, 2000, p. 8).

Ce qui vient confirmer l'importance du respect de la nature ainsi que du maintien de l'équilibre écologique.

Patel donne à mieux comprendre les enjeux environnementaux qui guettent la société. Loin d'être une écriture mineure, la bande dessinée de Patel montre comment elle a diversifié les formes narratives traditionnelles à travers les illustrations de Joëlle Maëstracci "tout en volutes et en arabesques avec des couleurs lumineuses " (Cassiau-Haurie, Africultures, le monde en relation). Comme toute image est polysémique, les données visuelles sont très importantes car elles agissent comme des signes importants à décrypter. L'échelle des plans et l'omniprésence du bleu (pour La toile bleve) donnent l'impression au lecteur qu'il est dans l'océan ou près de lui. Nous tenterons d'analyser, dans la partie qui suit, ces espaces qui sont porteurs de significations précises, et notamment ceux qui mettent en valeur la couleur locale.

\section{Quand Shenaz Patel valorise la couleur locale}

Dans son article "Qu'est-ce que l'exotisme? », Jean-François Staszak définit le mot exotisme comme ce qui caractérise « un lieu comme lointain (...) le bizarre » (Staszak, 2008, p. 8), en mettant en avant le fait que c'est à la fois le propre d'un objet (on parle de bois exotique) et d'un sujet (on parle de l'exotisme de Gustave Flaubert). Cependant, tout ce qui est exotique l'est nécessairement pour l'étranger et non pour l'habitant du pays. Or, Shenaz Patel, dans Le bestiaire mauricien (2016) - recueil de neuf histoires dont l'essence et les sujets principaux sont les animaux vivant à l'île Maurice - donne une nouvelle dimension au terme exotisme, même s'il s'agit non de ce que précise Patel mais de ce que nous ressentons en tant que lecteur. Ce qui ressort, à la lecture de cette œuvre joliment illustrée par Emmanuelle Tchoukriel, serait un idéal esthétique, tant dans les histoires présentées que dans l'illustration elle-même. Si l'exotisme reflété dans les noms propres issus du contexte mauricien comme " bébêtes-ciseaux", " paille-en-queve ", " tangue ", " tec-tec » ou encore "l'ourite" est au centre de l'entreprise littéraire de Patel, on pourrait percevoir, à travers cela, comment le recours à l'exotisme passe par une auteure qui jove avec les mots. Cela pourrait être un remède aux maux causés à l'environnement; on pourrait également y voir une quête existentielle et un idéal esthétique qui relèvent des idéaux de l'auteure. Patel instruit l'enfant-lecteur sur la culture de la désagrégation par l'arrivée des grands développements :

Entre 1880 et 1925, l'Extrême-Orient représente, pour les poètes et les écrivains, un certain idéal esthétique (...). Le processus de création littéraire laisse apparaître deux modes opposés d'idéalisation. Les voyages entrepris avec enthousiasme finissent par la désillusion provoquée par le choc avec des cultures en désagrégation, ce qui contribue à perpétuer les clichés négatifs ou bien, pour certains écrivains, à vouloir conserver l'image d'un Extrême-Orient traditionnel, immuable (Bernier, 2001, p. 47). 
Dans "Comment les bébêtes-ciseaux sauvèrent les bananes", Patel aborde le problème des "constructions d'hôtels et de maisons partout sur l'île " qui " avaient singulièrement détruit les forêts où ils (les paille-en-queve) s'abritaient d'habitude ॥ (Patel \& Tchoukriel, 2016, p. 13). C'est ainsi que l'écriture de Patel fait preuve d'une efficacité littéraire par ses formes linguistiques empruntées au contexte local. II s'agit des mots composés comme " bébêtes-ciseaux " ou "paille-en-queve ", mais aussi des mots composés "doublés" comme "tec-tec ». Les "bébêtes-ciseaux" sont des insectes noirs en forme de ciseaux que l'on retrouve dans les régimes de bananes. Patel tente par ce biais de faire un renvoi au problème auquel les animaux font face en indiquant les maux qui expliquent comment certaines espèces seraient en voie de disparition. Le chercheur Gerardo Ceballos, de l'Institut d'Écologie de I'Université Nationale Autonome du Mexique, précise à ce sujet : "Nous avons perdu en 500 ans plus de 1000 espèces et en 100 ans, entre 300 espèces et 400 espèces. Le taux d'extinction des 100 dernières années est cent fois plus important que celui des 2 derniers millions d'années. Les vertébrés qui ont disparu lors des cents dernières années auraient dû disparaître en 10000 ans $\|^{3}$.

La représentation de la violence environnementale sur l'île Maurice sera plus subtile, mais non moins insidieuse. La régression voire la disparition des espèces locales, causée par la déforestation, la pollution ou l'urbanisation est le problème majeur auquel l'auteure s' attaque, mais elle le fait de manière ludique. Son écriture caustique est si subtile que cela n'est pas manifeste, l'auteure ayant l'art de mêler à toute forme de critique, une forme d'humour imagé. En effet, dans « Le mystère de l'ourite à dix pattes ", Patel explique la raison pour laquelle la pieuvre (l'ourite en créole) a dix pattes (à travers un " petit bigorneau ॥ qui s'interroge sur cette disparition) ; elle avait besoin de deux de plus afin de « mieux danser avec deux étoiles de mer rencontrées en chemin» (Patel \& Tchoukriel, 2016, p. 19)! Dans la triste mais belle histoire du "Pacte du papillon et du gecko », la survie de la liane Rousseau ne se fait que par le gecko qui la pollinise car :

depuis la disparition de l'oiseau à lunettes, il (le papillon) se retrouve à devoir tout faire tout seul, toutes les plantes qu'ils partageaient auparavant, elles le sollicitent de partout. II ne sait plus où donner des ailes (Patel, 2016, p. 26).

Certes, c'est de manière imagée que le vocabulaire donne vie aux histoires écrites par Shenaz Patel. D'abord, notons que les contes sont divisés selon les éléments terremer-air. C'est ainsi qu'il y a « trois animaux marins, trois créatures terrestres et trois bêtes qui volent "4. Dans "Des tec-tec dans le sable ", Shenaz Patel fait un clin d'œil aux coquillages, que les Mauriciens trouvent facilement le long des plages locales. L'histoire débute en précisant comment, à un certain moment de l'histoire, "les tectec vivaient sur les rochers ", harmonieusement avec «les bigorneaux, les cônes, les petites coquilles-bonheur, les grosses porcelaines" (Patel \& Tchoukriel, 2016, p. 29). Ainsi, il y a comme un rappel de l'anthropomorphisme (par le biais des animaux étranges et parlants comme dans Alice au pays des merveilles de Lewis Carroll), Iorsque, chez Patel, les coquilles commencent à manifester leur colère.

\footnotetext{
3 Citation d'après Gerardo Ceballos dans «Biodiversité : accélération de la disparition d'animaux sauvages II, de Maïwen Bordron, 30/10/2018, https://www.franceculture.fr/ecologie-etenvironnement/biodiversite-acceleration-de-la-disparition-danimaux-sauvages [17/01/2021].

4 «L Le bestiaire mauricien : cherchez la petite bête », lexpress.mu [20/02/2017].
} 
On pourrait faire remonter l'expression "couleur locale " jusqu'à l'époque du classicisme (Brunot dans Kapor, 2003, p. 1). Elle serait empruntée au vocabulaire lié à la peinture de la fin du XVIIe siècle. Si l'expression en français lui donne un statut particulier, Vladimir Kapor fait remarquer que, dans le domaine anglophone, le « local color ", reste en général "privé de connotations péjoratives" (Kapor, 2003, p. 10). Patel éveille le sens de la couleur locale chez l'enfant afin que ce dernier se rende compte, en devenant adulte, de l'importance de la survie des espèces locales (surtout celles en voie de disparition). Il serait intéressant, ici, de souligner l'importance que Patel accorde à l'animal. L'animal turn est une philosophie qui est en vogue dans les universités. En reprenant une idée de Baratay, Débarre, Baloge, Klimpe, LambertzPollan, Tochachi et Seitz précisent que «dans I'histoire occidentale, l'animal a été convié, servant tantôt de miroir grossissant des forces et des faiblesses humaines, tantôt de lieu d'une irréductible altérité " (Débarre et al., 2013). Les auteurs poursuivent leur argumentaire en faisant référence à Gilles Deleuze qui, en 1988, démontre que les animaux domestiques avaient tendance à perdre de leur animalité. Cependant, et comme le précise Baratay en 2003, la volonté de l'homme à vouloir dominer l'animal et à utiliser cet être à des fins particulières a fait que l'animal a perdu le statut qu'il avait: c'est ainsi que s'est érigée une barrière entre l'animal et l'homme. L'urbanisation accentuera, elle aussi, cette démarche (Baratay, 2003, pp. 24-42). La mise en narration de l'animal chez Patel, à travers l'animal qui parle, n'est pas sans rappeler les fables de la Fontaine. Notons par ailleurs que si au Moyen Âge comme à l'époque romantique, on attribuait volontairement des caractéristiques humaines aux animaux - notamment à travers la parole et par le biais des figures comme la personnification ou la prosopopée -, c'est le nature writing qui a défini les nouveaux paramètres des textes à caractère environnemental (Lawrence Buell). Dans sa thèse de doctorat, Julien Defraeye aborde l'origine du nature writing comme suit :

Le nature writing ${ }^{5}$, que l'on peut circonscrire au contexte étatsunien du début du XIXe à la première partie du XXe siècle, recouvre, si nous nous en tenons à une définition liminaire, des écrits majoritairement non fictionnels, de prose ou de poésie, mêlant souvent descriptions de la nature et autobiographie. Ceux-ci héritent supposément des histoires naturelles popularisées au XVIIII siècle, qui présentaient des descriptions méthodiques de la nature - faune et flore principalement -, à la différence que celles-ci excluaient l'humain et ne proposaient pas de mise en récit, préférant le visuel des planches de botaniques au texte. Mentionnons la prédominance du nature writing en Allemagne et en France par l'impact des voyages d'Humboldt, et, dans le monde anglophone - puisque le nature writing est avant tout un genre de langue anglaise -, de Gilbert White (1720-1793), ornithologue et naturaliste anglais, qui étudie et reproduit sur le papier plus de 400 espèces de plantes et d'animaux dans le comté de Hampshire et le Sussex entre 1768 et 17936. William Bartram (1739-1823), à la suite de ses explorations des colonies américaines en 1773 - vingt ans avant Humboldt publie nombre de ses illustrations botaniques7 (Defraeye, 2019, p. 34).

\footnotetext{
${ }^{5}$ Consulter Mabey, R. (1995). The Oxford Book of Nature Writing. New York: Oxford University Press.

6 Consulter White, G. (1789). The Natural History and Antiquities of Selborne with Observations on Various Parts of Nature and the Naturalist's Calendar. London: Benjamin White.

7 Consulter Bartram, W. (1791). Travels through North and South Carolina, Georgia, East and West Florida, the Cherokee Country, the Extensive Territories of the Muscogulges or Creek Confederacy, and the
} 
Pour Patel, le nature writing passe par la métaphore animalière qui est utilisée afin de faire passer un message sur l'environnement, voire sur les animaux endémiques de l'île. Par exemple, c'est de manière poétique qu' ' [u]ne histoire de chauve-souris » est racontée. Le message est que le "petit rat musqué " (Patel \& Tchoukriel, 2016, p. 34) fait le choix de perdre la vue à la suite d'une demande auprès de l'Esprit des Animaux auquel il s'adresse pour pouvoir s'envoler. Il y a un jeu de miroirs en ce sens que la race humaine pourrait s'identifier au rat musqué, un peu à la manière d'une métamorphose ou d'une incursion dans le monde de la thérianthropie. De la même manière, I'histoire " Le voyage du courtpas » est une critique véhiculée par l'analogie animale qu'est le courtpas (ou l'escargot). Patel fait ici la caricature des personnes qui souhaitent aller vite en besogne et finissent par se faire mal, un peu comme le " [p] etit escargot » qui, par curiosité, décide d'aller « voir ce qu'il y a de l'autre côté du jardin» (Patel \& Tchoukriel, 2016, p. 38), et, n'ayant "jamais vu une pente » dégringole pour finir sa course en craquant sa coquille. Le message peut, de plus, convoquer un imaginaire mythique, reprenant le thème de la cosmogonie biblique présent dans "un amour d'étoile de mer» où l'histoire semble débuter comme la Genèse par le récit de la naissance des étoiles : "Au début, au tout début du monde, il y avait le Soleil, la Lune, et il y avait la planète Mer » (Patel \& Tchoukriel, 2016, p. 41). De I'histoire d'un paille-en-queve déchu de sa splendeur dans " Le vol du paille-enqueve » (Patel \& Tchoukriel, 2016, p. 8), aux « bébêtes-ciseaux » qui sauvent la vie des bananes, en passant par "les tec-tec » qui se bagarrent avec d'autres espèces de coquillages, les connotations variées réitèrent la représentation de ceux qui "habitent" les trois espaces "terre-mer-air », afin de mieux mettre en exergue ces figures incontournables de la « couleur locale».

Alors que la couleur locale fait partie intégrante des albums de Patel, nous avons été imprégnés de la complexité poétique de la nature. En ce sens, l'écopoétisation patelienne réinterroge notre rapport à l'environnement, et donne à réfléchir au fait même que l'esthétique scripturale de l'auteure est en elle-même une forme d'écologie.

\section{L'histoire en bande dessinée comme véhicule du savoir}

Les deux albums que nous avons préalablement étudiés semblent plus adaptés à des jeunes enfants qu'on souhaite sensibiliser aux problèmes environnementaux et initier à la notion de couleur locale. Il y a malgré cela une différence marquante entre la BD et les deux tomes de la bande dessinée Histoire de Maurice (T1 et T2). En effet, cette collaboration d'écriture avec Jocelyn Chan Low donne aux jeunes et aux moins jeunes la possibilité de comprendre deux périodes de l'histoire de Maurice.

II s'agit de deux périodes qui sont délimitées, désignées et intitulées comme suit : la première, de 1598 à 1767, «Premiers pas de la colonisation d'une île-carrefour » et la deuxième, de 1767 à 1885, "L'expansion d'une colonie très convoitée ». Notons, par ailleurs, que le tome 2 semble encore plus méticuleux en termes de recherches que le premier. Dans les deux cas, deux jeunes (une fille et un garçon), sont à la bibliothèque Carnegie, sise à Curepipe, au centre de l'île Maurice. Le garçon est en pleine lecture de La Machine à remonter le temps de H. G. Wells quand la fille lui demande : «Si tu pouvais voyager dans le temps, tu choisirais d'aller où? " (Patel et

Country of the Chactaws. Containing an Account of the Soil and Natural Productions of Those Regions; Together with Observations on the Manners of the Indians. Philadelphia: James \& Johnson. 
al., 2017a, p. 3), ce à quoi ce dernier répond: «Dans le futur en l'an 3000 ». La fille, quant à elle, ironise en disant qu'elle irait « dans le passé de [son] île » (Patel et al., 2017 a, p. 3), et précise que, le même matin, on a parlé de la notion de citoyenneté en classe. Un certain Simon Van de Stel surgit de la tablette tactile de la fille pour lui expliquer comment il avait été le premier citoyen métis de l'île, né à Maurice «le 14 octobre 1639 " d'un père Hollandais et d'une Indienne (elle-même "fille d'une esclave indienne libérée ") (Patel et al., 2017a, p. 4) et qu'il allait, par la suite, être nommé «Commandeur du Cap ».

Ce sera sous un angle particulier que Patel et Chan Low articuleront leurs propos, c'est-à-dire à travers la figure d'analepse. En effet, l'on ressent chez les deux auteurs, une volonté marquée de mettre le métissage au centre de leur écriture. De ce début de récit, l'île Maurice, comme on le démontrera par la suite, est parmi les pays qui ont subi le plus de changements tant économiques, sociaux qu'ethniques, et, de ces mutations sont nées certaines spécificités dont fait partie le métissage. Les deux tomes, dont la mise en images est d'un style réaliste sont signés Laval Ng et de Christophe Carmona pour le premier tome et Christophe Carmona pour le deuxième. Les albums sont en majeure partie une forme de transposition de la réalité abordant les brassages ethniques auxquels ont fait face l'ensemble de la communauté mauricienne. Ce brassage naît de ce "lieu-carrefour, au croisement des mers, des civilisations, des cultures, des rêves d'ailleurs, des désirs de conquêtes, des projets de création des hommes ॥ (Patel et al., 2017a, p. 5) qu'a toujours représenté l'île Maurice. Cette idée de syncrétisme et d'une forme de médiation de la minorité identitaire se traduit à travers un "entre-deux " du temps et de l'espace, bien que les deux jeunes rappellent que le présent se situe dans un contexte différent.

En ce sens, la bande dessinée qui relate ces événements - elle-même une forme hybride qui laisse les mots et les images raconter des histoires - permet, de manière plus rapide, aux jeunes de retenir le contenu qui s'inscrit soit comme un art séquentiel soit comme une forme moderne de narration figurative (Fresnault-Dervelle, 9). Comme le précise Anna Bobińska:

La construction du sens dans le récit bédéistique repose donc sur l'interaction entre ce qui est visuel (dessin, trait, graphisme, perspective, symétrie, cadrage, couleurs et blancs, gestuelle des personnages, bruits etc.) et ce qu'appartient aux codes discursifs ou littéraires (intrigue, conception, expression des mouvements et des émotions, grammaire, syntaxe etc.) (Bobińska, 2015, p. 314).

L'on comprend comment les planches et les bulles, considérées ensemble, renvoient déjà à une forme de métissage. "L'encapsulation» (Eisner, 2009, p. 45) restitue pleinement l'Histoire de Maurice, du "cartographe vénitien Alberto Cantino" (Patel et al., 2017b, p. 6) qui « fait figurer sur son planisphère "les "trois îles" découvertes par les hommes de Vasco da Gama" ॥ (Patel et al., 2017a, p. 6), jusqu'aux Hollandais qui baptisent l'île "T'Eylandt Mauritius Van Nassau " (Patel et al., 2017b, p. 8), le 21 septembre 1598, en passant par les dodos et les ébéniers qui deviendront les souffredouleurs de ces premiers colons (Patel et al., 2017a, p. 10). Les cases muettes, comme celles qui décrivent le luxuriant décor lorsque les Hollandais découvre l'île, ainsi que ses dodos (Patel et al., 2017a, p. 9-10), offrent un mariage surprenant entre la dimension visuelle et l'approche techniciste des tableaux. Les codes sémiotiques linguistique et figuratif (par le dialogue et la représentation des actions 
respectivement), à travers leur structuration, permettent de mieux s'approprier le langage de la bande dessinée. Eł ce sera de manière à assurer la coordination dans les idées, tout en respectant les données historiques de manière chronologique, que nous apprendrons, par exemple, que le «13 octobre 1601, les hommes de vaisseau Duifje trouvèrent à l'île Maurice un Français qui raconta avoir quitté l'Angleterre quelques années plus tôt sur un bateau qui fit naufrage "s et qu'il fut, très probablement "le premier "habitant" de l'île" (Patel et al., 2017a, p. 11), ou encore que le père du narrateur, Adrian Van der Stel, « [ramena] 106 esclaves (...) dans la baie d'Antongil à Madagascar " (Patel et al., 2017a, p. 13). Nous apprendrons également comment les Hollandais formaient une population « peu homogène » (Patel et al., 2017a, p. 16) ou encore comment l'esclave Anna du Bengale mit le feu à la loge du Fort Hendrik (Patel et al., 2017b, p. 21), incendie auquel assista François Leguat, et qui fut relaté dans ses mémoires, puisqu'il assista à cet incendie (Patel et al., 2017b, p. 21). Thierry Groensteen, dans Système de la bande dessinée, précise comment des cinq « types de détermination" de la bande dessinée, "les propriétés globales sont la super ordination, la coordination, la subordination et la pré ordination $»(p .7)$, ce qui démontre à quel point « la vignette [qui est] l'unité de base du langage de la bande dessinée " possède une importance supérieure aux personnages eux-mêmes. Ce qu'Eisner qualifie de juxtaposition de mots et d'images permet, d'une part, de décoder des formes graphiques, et, d'autre part, de les encoder. On encode la représentation de l'histoire de Maurice afin de produire un impact particulier sur le lecteur: I'arrivée des "forçats chinois" (Patel et al., 2017b, p. 23), celle des pères Lazaristes à la paroisse de Notre Dame des Anges à Vieux-Grand-Port (Patel et al., $2017 a$, p. 28) - quand la Compagnie des Indes voulait répandre la religion chrétienne - l'arrivée, en 1756, des «femmes esclaves venues de la côte Malabar, Bengale, Pondichéry " (Patel et al., 2017a, p. 32), ou encore ce que les esclaves malgaches ont laissé comme héritage comme la "culture du brûlis qui améliore la culture, l'introduction de la pêche au casier " (Patel et al., 2017a, p. 36). Le langage corporel des personnages, bien que ces derniers soient « secondaires" (Groensteen, op. cit.), ainsi que les expressions des visages, améliorent la narration graphique pour mieux atteindre le jeune public lecteur. Vers la fin du premier tome, il y a une référence à une visite au musée de Mahébourg, qui rappelle aux jeunes que ce musée recèle les "vestiges du Saint Géran» (Patel et al., 2017a, p. 45). Qu'elle soit dialogique ou récitative, qu'elle contienne des onomatopées ou encore des cartouches purement historiques, l'écriture adopte une conception scénaristique en osmose avec les bulles ; tantôt descriptive, lorsque, par exemple, on décrit une scène représentée sur un plan d'ensemble, tantôt narrative, lorsque le plan devient rapproché et se fait à travers un gros plan sur un personnage ou deux personnages qui dialoguent. Les modalités esthétiques (décor, couleurs, formes et angles de vue) permettent à une interprétation de se faire aisément. Toutefois, le trop grand flot d'information observé dans les deux tomes tendrait à alourdir quelque peu le mouvement de l'histoire. La fictionnalisation de l'histoire est une technique aboutie si les éléments présentés sont en équilibre. La mise en images semblerait plus éloignée de la réalité que l'approche historiciste puisque la bande dessinée de manière générale favorise de toute évidence cette posture.

Le deuxième tome, c'est-à-dire "L'expansion d'une colonie très convoitée ", est beaucoup plus référencé historiquement. Aussi parle-t-on du Chevalier de Tromelin (Patel et al., 2017b, p. 4) appelé à Maurice pour « développer Port-Louis » (Patel et al., 
$2017 b$, p. 4) et l'on présente l'île-carrefour comme l'île du commerce des esclaves (Patel et al., 2017b, p. 5). La trame narrative conserve le référent du tome 1, tout en étant moins catégoriel ou classificatoire. Ainsi attribue-t-on une importance à la présence des "gens de couleur" (Patel et al., 2017b, p. 7). Nous voyons comment "beaucoup de femmes ayant eu des enfants avec leurs maîtres [finissent par] les déclar[er] comme filleuls, et (...) achètent des terrains pour garantir leur avenir " (Patel et al., 2017b, p. 10). On n'hésite pas non plus à rappeler comment Napoléon rétablit l'esclavage dans les colonies (Patel et al., 2017a, p. 15) et comment, subséquemment, les « gens de couleur » ne bénéficieront plus de la protection auparavant assurée par la filiation (Patel et al., 2017b, p. 16). Le deuxième tome accordera une importance particulière à la bataille du Vieux-Grand-Port, moment phare dans I'histoire de Maurice, cette bataille étant « inscrite sur l'Arc de Triomphe à Paris comme la seule victoire navale de Napoléon sur les Anglais " (Patel et al., 2017b, p. 24) ${ }^{8}$. On peut alors estimer que l'île Maurice, ayant vécu l'arrivée des personnes de multiples points du globe, aura connu une véritable transculturation. L'entrecroisement de ces peuples a donné lieu à une conception symbolique de l'île, qui a elle-même bourgeonné en une île-carrefour où cohabitent différentes catégories et sous-catégories ethniques. Patel n'a eu de cesse de rappeler l'hybridité qui a marqué l'histoire de Maurice et nous observons que la mixité ethnique qui, d'ailleurs, débute l'histoire du premier tome, est une fois de plus mise en avant dans le deuxième tome, par exemple lorsque "Les Indiens se mettent à recréer leurs villages à Maurice. Le système de castes est reproduit. La société se referme ॥ (Patel et al., 2017b, p. 46). Parole débridée, identités menacées ou ouvertures à une diversité souhaitée, qui serait le signe d'une mobilité identitaire et d'un certain progrès?

\section{Conclusion}

Les œuvres de Patel que nous avons étudiées nous ont permis de confirmer un fait: l'écriture de cette auteure dépasse la forme première d'une écriture pour enfants et jeunes, cadres restrictifs généralement imposés à ces bandes dessinées. Les frontières semblent abolies. En abordant l'environnement, la couleur locale (dans le contexte mauricien), ainsi que I'histoire de Maurice, on comprend mieux comment Patel souhaite, en tant qu'auteure, insérer ses récits dans une forme réelle de la représentation. Le récit bédéistique réussit bien à Shenaz Patel, qui était déjà connue auparavant pour ses récits « traditionnels». Même si l'auteure elle-même précise ce qui suit : «L'écriture pour moi c'est une sonorité. S'il y a une conclusion pratique aux histoires, c'est sous-jacent parce que je n'aime pas que l'on me dise ce qu'il faut penser, ce qu'il faut croire " (L'Express, 20 février 2017), nous percevons tout de même dans son écriture une volonté de faire passer une leçon, une morale. Patel valorise la

\footnotetext{
8 Gravière, J. de la (1887). Les héros du Grand-Port. Revue des Deux Mondes. 84 (3), p. 108 :

«Le 27 août apparut à l'entrée du port la division Hamelin, composée de trois frégates et d'un brick : la Vénus, la Manche, l'Astrée et l'Entreprenant. Sommé de se rendre, le commandant de l'Iphigénie, le capitaine Lambert, reconnut l'impossibilité d'opposer à tant de forces réunies le feu de sa seule frégate : il nous remit, avec l'Iphigénie, le fort de la Passe. Le pavillon anglais n'y avait flotté que pendant quatorze jours. N'étions-nous pas en droit de nous écrier, comme Nelson après Aboukir : "Ce n'est pas une victoire, c'est une conquête? ॥. Jamais triomphe ne fut plus complet. Nous l'avions, il est vrai, payé cher. Les deux frégates et le Ceylan comptaient 150 marins et plusieurs officiers hors de combat.

Les Anglais ne nous ont pas souvent donné de ces joies-là. Ce n'était pourtant pas la dernière que nous réservaient les mers de l'Inde ॥.
} 
couleur locale dans ses écrits en vue de combattre le phénomène des extinctions d'espèces. Finalement, l'histoire minutieusement racontée et retranscrite en images vise à rendre le passé accessible à tous.

\section{Bibliographie}

(20 février 2017). Le bestiaire mauricien: cherchez la petite bête. Lexpress. https://www.lexpress.mu/article/300634/contes-contemporains-bestiairemauricien-cherchez-petite-bete [13/01/2021].

(30 avril 2017). Cessons de nous mentir sur notre Histoire. Interview réalisé par Fabrice Aquilina. Lexpress. http://www.lexpress.mu [13/01/2021].

Baratay, E. (2003). Et l'homme créa l'animal. Histoire d'une condition. Paris: Odile Jacob.

BARATAY, E. (2012). Le Point de vue animal. Une autre version de I'histoire. Paris : Seuil.

BARTRAM, W. (1791). Travels through North and South Carolina, Georgia, East and West Florida, the Cherokee Country, the Extensive Territories of the Muscogulges or Creek Confederacy, and the Country of the Chactaws. Containing an Account of the Soil and Natural Productions of Those Regions; Together with Observations on the Manners of the Indians. Philadelphia: James \& Johnson.

BECKETT, S. L. (2008). Crossover fictions. Oxford \& New-York: Routledge.

BENSE, M. (1954). Aesthetica I. Stuttgart : Deutsche Verlagsanstalt.

BENSE, M. (1956). Aesthetica II. Krefeld and Baden-Baden : Agis-Verlag.

BENSE, M. (1958). Aesthetica III. Krefeld and Baden-Baden : Agis-Verlag.

BENSE, M. (1960). Aesthetica IV. Krefeld and Baden-Baden: Agis-Verlag.

BERNIER, L. (2001). Fin de siècle et exotisme. Le récit de voyage en Extrême-Orient. Revues de Littérature comparée, 297 (1), pp. 43-65.

BoBIŃSKA, A. (2015). Esthétique de la bande dessinée : métissage entre le texte et l'image. Svět literatury - Časopis pro novodobé literatury. http://cejsh.icm.edu.pl/cejsh/element/bwmetal .element.desklight-377373bfcd6a-47ce-88f9-918c6abe0064/c/anna_bobinska_313-321.pdf [03/01/2021].

BOURQUE, A. (2000). Les changements climatiques et leurs impacts. Vertigo, 2 (1), https://journals.openedition.org/vertigo/4042 [18/05/2021].

BRUNOT, F. (1968). Histoire de la langue française, 4 vol. Paris : A. Colin.

CASSIAU-HAURIE, C. (2011). La toile bleve, comme un point d'orgue à la montée de la littérature de jeunesse mauricienne. Africultures, le monde en relation. https://africultures.com/la-toile-bleve-comme-un-point-dorgue-a-la-monteede-la-litterature-de-jeunesse-mauricienne-10165 [15/01/2021].

DÉBARRE, S., Baloge, M., Klimpe, H., Lambertz-Pollan, R., Pourahmadali Tochahi, M., \& Seitz, A. (2013). La condition animale : Places, statuts et représentations des animaux dans la société. Trajectoires, 7. https://journals.openedition.org/trajectoires/1247 [06/10/2021].

DEFRAEYE, J. (2019). Perspectives écopoétiques dans le récit québécois contemporain. Ontario. https://uwspace.uwaterloo.ca/handle/10012/14689 [18/05/2021].

EISNER, W. (2009). Les clés de la bande dessinée 1. L'Art séquentiel. Paris: Éditions Delcourt.

Fresnault-Deruelle, P. (2009). La bande dessinée. Paris : Armand Colin.

GLOTFELTY, C. (1996). Introduction: Literary studies in an age of environmental crisis. In GLOTFELTY, C. \& FROMM, H. (ed.), The ecocriticism reader, Athènes \& Londres : University of Georgia Press, pp. 15-37. 
Graviere de LA, J. (1887). Les héros du Grand-Port. Revue des Deux Mondes. 84 (3), pP. 101-123.

GroensteEn, T. (1999). Système de la bande dessinée. Paris : PUF.

KAPOR, V. (2003). Exotisme et couleur locale - essai d'une analyse contrastive des champs sémantiques respectifs. In Proceedings, France and the Exotic. University of Birmingham. http://hdl.gandle.net/11343/34666 [06/11/2021].

LIMIS, S. \& VAN EYNDE, L. (2002). Littérature et savoir(s). Bruxelles: Presses de l'Université Saint-Louis. http://www.openedition.org/6540 [15/01/2021].

MABEY, R. (1995). The Oxford Book of Nature Writing. New York : Oxford University Press.

PATEL, S. \& MAËSTRACCI, J. (2010). La toile bleve. Cassis : Editions Vilaz Métis.

PATEL, S. \& TCHOUKRIEL, E. (2016). Le bestiaire mauricien. Maurice : Atelier des Nomades.

Patel, S., Chan Low, J., NG, L. \& Carmona, C. (2017a). Histoire de Maurice. 1598-1767, Premiers pas de la colonisation d'une île-carrefour, vol. 1. Strasbourg : Éditions du Signe et Cassis : IPC.

Patel, S., Chan Low, J., NG, L. \& Carmona, C. (2017b). Histoire de Maurice, 1767-1885, L'expansion d'une colonie très convoitée, vol. 2. Strasbourg : Éditions du Signe et Cassis : IPC.

StASZAK, J.-F. (2008). Qu'est-ce que l'exotisme?, Le Globe. Revue genevoise de géographie, 148 (1), pp. 7-30.

White, G. (1789). The Natural History and Antiquities of Selborne with Observations on Various Parts of Nature and the Naturalist's Calendar. London: Benjamin White.

XINGJIAN, G. (2010). L'environnement et la littérature. Écrire aujourd'hui. Discours d'ouverture au Forum international du PEN Club Tokyo, le 26 septembre 2010. Denis Molcanov (trad.), Poésie, 134, pp. 3-15. 